\title{
Ciberantropología del periodismo en red. Enfoques teóricos y propuestas metodológicas para el estudio de la audiencia digital
}

\author{
Cyberanthropology of Network Journalism. \\ Theoretical Approaches and Methodological Proposals for the Study of the \\ Digital Audience
}

\author{
Jesús Miguel Flores Vivar \\ Universidad Complutense de Madrid, Madrid, España \\ Contacto: jmflores@ucm.es \\ https://orcid.org/0000-0003-1849-5315
}

\begin{abstract}
Resumen
El artículo analiza la inclusión de las neurociencias como la antropología y la etnografía en el estudio de las audiencias en internet. Estas disciplinas, al ser aplicadas en entornos digitales, derivan en nombres como ciberantropología, antropología digital y etnografía virtual. Estas adquieren relevancia en los procesos comunicativos de la gente mediada por ordenador, y están influenciadas por las nuevas formas narrativas, proponiéndose un enfoque más transversal y multidisciplinario en el estudio de usuarios con el objetivo de conocer y detectar las necesidades de los mismos, así como conocer con profundidad el fenómeno de masas que se alinean en las redes sociales utilizando diferentes dispositivos móviles. Finalmente, se sugieren algunos pasos adicionales para la articulación de una antropología del ciberperiodismo, explorando las implicaciones de las disciplinas tratadas como estrategias innovadoras en el estudio del periodismo y comunicación.
\end{abstract}

Palabras clave: Internet; Etnografía; Audiencia; Antropología; Ciberperiodismo

\begin{abstract}
Article analyzes the inclusion of neurosciences such as Anthropology and Ethnography in the study of online audiences. These disciplines, when used in digital environments, derive in names such as cyberanthropology, digital anthropology or virtual ethnography. These acquire relevance in the communicative processes of the people mediated by computer, influenced by the new narrative forms, proposing a more transversal and multidisciplinary approach in the study of
\end{abstract}


users with the aim of knowing and detecting the needs of the same, as well as, knowing in depth the phenomenon of masses that are aligned in social networks using different mobile devices. Finally, some additional steps are suggested for the articulation of Anthropology for Cyberjournalism, exploring the implications of the disciplines treated as innovative strategies in the study of journalism and communication.

Keywords: Internet; Ethnography; Audience; Anthropology; Cyberjournalism

\section{Los primeros tramos}

El periodismo y los medios de comunicación han sido sacudidos por el tsunami de la crisis económica mundial y por la crisis estructural de los propios medios. Esta situación ha obligado a tener que estudiar, explorar, investigar, analizar y reflexionar sobre nuevas teorías, nuevos axiomas y nuevos planteamientos con el objetivo de acrecentar el valor y la calidad del nuevo periodismo en internet. En este punto, es necesario, primero, recordar que el conjunto de los factores que componen el periodismo sigue estando compuesto por los profesionales (quienes informan), los medios (por donde se informa) y la audiencia (a quien se informa); $\mathrm{y}$, segundo, abordar el estudio de estos elementos, principalmente, de la audiencia, desde una perspectiva más interdisciplinaria. Como la audiencia de los medios ha sido estudiada desde la sociología, ciencia social que ha convivido con el periodismo y la comunicación, ahora corresponde estudiarla desde el ámbito de las humanidades, concretamente, desde la antropología, intentando comprender cómo se forman y mueven los grupos de personas (usuarios) en un nuevo escenario que tiene su base en las plataformas de internet y en la telefonía móvil. Para ello, es necesario profundizar en los perfiles de usuarios que buscan y forman nuevos espacios virtuales, indagar cuáles son los intereses perseguidos y qué es lo que la propia internet ofrece. Según Vázquez (2008), "tal vez nos llevemos una sorpresa, y veamos con asombro de qué manera este nuevo medio puede ayudar a desarrollar nuevas etapas y vivencias". Y la antropología no puede dejar pasar por alto este nuevo escenario. Para el autor, "Internet se convierte en 
un espacio antropológico, un nuevo escenario con unas reglas propias. La ciberantropología” (2008, p. 2).

\section{Antecedentes}

El trabajo de la ciencia cognitiva ha mostrado que muchos rasgos de la cognición humana son universales, posiblemente porque se basan en una arquitectura neural compartida, aun cuando la expresión de tal arquitectura esté significativamente modulada por fuerzas culturales (Brown, 2010, p. 36). Por ello, es importante conocer a los diferentes colectivos que inundan la red. Desde usuarios anónimos hasta expertos navegantes que trabajan para las diferentes organizaciones empresariales, pasando por una audiencia "prosumidora" (acrónimo de productor + consumidor) de los medios.

Pese a la importancia actual de los bloggers y de las tecnologías emergentes de la información, son muy escasos y residuales los estudios de fundamento socioantropológico realizados sobre este colectivo de personas, cuyo rasgo más característico es que forman parte de una sociedad con cultura de red o cibercultura. Sin embargo, otro colectivo que crece en importancia y que posee cultura de red lo conforman las masas ingentes de usuarios de los medios, quienes a través de redes sociales o comunidades virtuales buscan e intercambian información de interés en la web. Por tanto, conocer la cultura o subcultura de red que conforman las comunidades virtuales es el leitmotiv de este estudio. En este contexto, es necesario entender el nuevo concepto de cultura digital que trasciende del propio origen de la palabra cultura que, según Marvin Harris (2014, p. 28) "es el conjunto aprendido de tradiciones y estilos de vida, socialmente adquiridos, de los miembros de una sociedad, incluyendo sus modos pautados y repetitivos de pensar, sentir y actuar". Así, la cultura de red de los usuarios navegantes del ciberespacio debe estudiarse desde una perspectiva más etnográfica y antropológica, ya que - en palabras de Harris - forma parte de "ese todo complejo que comprende conocimientos creencias, arte, moral, derecho, costumbres, y cualesquiera otras capacidades y hábitos adquiridos por el hombre en tanto que miembro de la sociedad”. Para sir Edward Burnett Tylor, citado por Harris (2014, p. 28), “[1]a 
condición de la cultura en las diversas sociedades de la humanidad, en la medida en que puede ser investigada según principios generales, constituye un tema apto para el estudio de las leyes del pensamiento y la acción humanos".

En este escenario, es importante comprender el significado de la subcultura que acarrea internet, dado que la cultura de una sociedad tiende a ser similar en muchos aspectos de una generación a otra. En parte, esta continuidad en los estilos de vida se mantiene gracias al proceso conocido como endoculturización. Según Harris (2014, p. 30), la endoculturización es una experiencia de aprendizaje parcialmente consciente e inconsciente a través de la cual la generación de más edad incita y obliga a la generación más joven a adoptar los modos de pensar y comportarse tradicionales. Es decir, adoctrinar en una cultura que han conocido las distintas generaciones y que intentan no cambiar; ello ha dado lugar al llamado etnocentrismo, en donde solo lo conocido es bueno y lo que no se conoce o lo extraño es malo. Mas, en la actualidad, y en el plano digital, esto no siempre ocurre así, ya que los jóvenes, conocidos ya como la generación App,

[...] construyen sus propias experiencias desde su concepción inicial del mundo digital, formando sus propias comunas virtuales a través de los diferentes medios sociales. Por otra parte, los antropólogos culturales sienten curiosidad por las diferencias culturales. Algunos, no obstante, han ido más lejos y adoptado el punto de vista conocido como relativismo cultural, con arreglo al cual toda pauta cultural es, intrínsecamente, tan digna de respeto como las demás.

(Harris, 2014, p. 30)

Así, las comunidades de usuarios poseen una cultura digital que debe ser tenida en cuenta cada vez más, puesto que es una cultura a donde se ha trasladado gran parte de nuestras vivencias. En tal sentido, el enfoque antropológico en el estudio de las comunidades virtuales debe ser más aplicado.

Para Harris (2014, p. 489), el núcleo de la antropología aplicada consta de investigaciones encargadas por organizaciones públicas o privadas en espera de conseguir objetivos prácticos de interés para esas organizaciones. Aunque, en la 
mayoría de casos, la antropología aplicada es realizada por antropólogos culturales, eso no quita para que otros especialistas antropólogos de la física, arqueología o lingüística tengan sus propios aspectos aplicados. Entre esas organizaciones se cuentan departamentos y organismos federales, estatales, locales e internacionales, aunque también, las organizaciones privadas, como fundaciones y empresas, se suman en el uso de la antropología aplicada. En dicho contexto, las organizaciones de noticias pueden hacer uso de la antropología aplicada. Por ejemplo, en el estudio de las redes sociales, ya que no se trata de estudiar el software y tampoco cómo se dinamiza una red social, si no lo que interesa es conocer el estado de pensamiento y opinión de las personas usuarias de las redes sociales virtuales.

La digitalización no sólo ha evolucionado el mundo de las telecomunicaciones, sino otros muchos aspectos de la vida de las personas, algunos de ellos tan importantes que se habla de un nuevo estilo de vida digital. Hemos pasado, como indica el subtítulo del libro de José B. Terceiro, "Del Homo Sapiens al Homo Digitalis". O tal vez a un digitalismo que lo afecta todo (Cremades, 2001, p. 8).

La información se ha constituido en el bien de consumo y motor económico más preciado de la sociedad actual cada vez más digital. Ha venido a sustituir en importancia a las materias primas naturales, $y$, concretamente, a la principal fuente de energía: el petróleo. La estructura económica de esta nueva sociedad de la información ha propiciado el desarrollo de nuevos sectores productivos cada vez más pujantes como los medios de comunicación, el ocio, el turismo y otros muchos relacionados directamente con las nuevas tecnologías, como la informática, la microelectrónica, la robótica o la biotecnología (Cremades, 2001, p. 16).

En este esquema, la lectura de los discursos que han acompañado el nacimiento de los nuevos medios de comunicación produce a veces la impresión de que la historia tartamudea (Flichy, 2001, p. 11), por lo que las Casandras (en referencia a Casandra, la hija de Príamo y de Hécuba, que predijo la destrucción de Troya) creen ver en los nuevos medios digitales una amenaza para la cultura o las libertades del ciudadano o, simplemente, la desaparición de modelos de negocio de la industria de la información y comunicación.

En este contexto queda claro que internet genera una movilización social. 
Esta cuestión de movilización de los individuos en el seno de las tecnologías de la información y comunicación fue estudiada ya en un campo más restringido: el de la introducción de las primeras máquinas de tratamiento de la información (máquinas de escribir, de calcular) del siglo XIX. Joanne Yates (1994) demostró que ni el modelo de la coordinación a través de lo escrito ni el uso de estas nuevas máquinas se habría podido difundir si no hubiera habido un clima de mediación y de inclinación o adaptación al cambio.

Según Flichy (2001, p. 13), los usos de internet están mucho más diversificados. Una de las grandes dificultades del estudio de internet como plataforma de comunicación viene de su complejidad. Unos lo analizan como un nuevo medio de comunicación, otros como un instrumento de comunicación interpersonal o como un nuevo sistema de organización de empresas, y aun otros como un dispositivo de intercambio comercial. Todas estas facetas de internet raras veces son estudiadas de forma simultánea y, sin embargo, los individuos están presentes al mismo tiempo en diversas escenas de internet y las justificaciones de sus compromisos son globales. En tal sentido se puede decir que, por analogía con la expresión de Max Weber, hay que estudiar el espíritu de internet. Más aún, ahora corresponde estudiar lo virtual-real de las comunidades que conforman ese "espíritu” de la red.

Para Pierre Levy, citado por Flichy (2001, p. 14) "estamos pasando de una humanidad a otra", estamos frente a una mutación antropológica tan importante como la del neolítico. En esta línea, nos atrevemos a afirmar que atravesamos un proceso de cambio y transformación tan importante como fue el pasar de la Edad Media a la Edad Moderna y, como todo proceso de cambio, este se viene realizando en un espacio-tiempo que se ve en las generaciones de personas que han nacido bajo determinado hito tecnológico. Un ejemplo de ello viene dado por la coloquial frase de jóvenes que han nacido en paralelo al origen de Google en 1998, que preguntan a sus progenitores: "si no tenías Google, ¿cómo encontrabas la información?". Esta es la “Generación App” que conforma la cibercultura. 


\section{Objetivos}

La presente investigación surge de la reflexión sobre la dialéctica entre cuestionamiento y sumisión a los contenidos mediáticos por parte de los usuarios, consumidores y productores que conforman la nueva audiencia digital. Es ampliamente sostenido que los estudios de ciencia y tecnología han alterado radicalmente los enfoques pasados sobre la tecnología, desplazando así la concepción lineal del cambio tecnológico y posibilitando la apertura de sólidos programas de investigación que están dando como resultado una verdadera renovación teórica (Escobar, 2005 , p. 16). Esta renovación teórica hace que los procesos de comunicación que se dan, por ejemplo, a través de las redes sociales, se hayan transformado radicalmente.

Con estos antecedentes, el objetivo fundamental es estudiar la participación de la audiencia digital (usuarios de noticias) a través de dispositivos móviles y conectados a la red, con el fin de determinar el grado de cultura digital e influenciar a los usuarios, por ejemplo, en el acceso a las noticias e información publicitaria, así como lograr una mayor participación política y social. En segundo término, se persigue como objetivos específicos: i) estudiar y demostrar la viabilidad de la inclusión de la antropología y etnografía para conocer mejor los usuarios y costumbres de las personas que forman comunidades virtuales, y ii) estudiar la simbiosis de las redes sociales en internet y su relación con los nuevos productos narrativos y nuevos lenguajes para lograr una mejor influencia, por ejemplo, en la navegación de los sitios web de noticias, en la compra, en el voto de usuarios, descubriendo las necesidades de estos.

Para ello, se ha de recurrir al estudio de las neurociencias como la antropología y la etnografía. La inclusión de estas disciplinas del conocimiento, con el interés puesto en la inmersión en las comunidades de usuarios, dibujará un panorama que hará más rentable a las organizaciones de noticias y les permitirá dar un tipo de información de calidad contrastada a un público más alfabetizado digitalmente.

Facebook y Twitter son en la actualidad las redes sociales más utilizadas, con más de mil quinientos millones de usuarios activos la primera y más de mil millones de usuarios la segunda. Entendido el alcance de estos espacios de interacción y 
comunicación, es de especial interés para la etnografía y la antropología comprender el uso que los individuos dan a estas redes sociales a través de dispositivos móviles. Así, según la descripción de Sören Romero (2014), para la red social Facebook el acercamiento a las redes sociales por parte de la etnografía virtual se puede hacer desde distintos enfoques.

Según Escobar (2005, p. 9) el estudio de la cibercultura está relacionado particularmente con las construcciones y reconstrucciones culturales en las que las nuevas tecnologías están basadas y a las que a su vez ayudan a tomar forma. En tal contexto, y tomando como referencia dicha afirmación, el punto de partida de este cuestionamiento es la creencia de que las redes sociales representan una invención cultural en el sentido de que esta contribuye a formar un mundo nuevo. Distintos investigadores antropológicos coinciden en que toda tecnología emerge de unas condiciones culturales particulares y de forma concomitante ayuda a producir otras.

Para Cristine Hine (2004), hasta ahora "se ha dedicado mucho más esfuerzo a predecir un futuro revolucionario de internet que a investigar, en detalle, cómo se utiliza y de qué modos se incorpora a la vida cotidiana de las personas". Creemos, pues, que ha llegado el momento de dedicar los esfuerzos a investigar estos aspectos, que son cruciales para el periodismo y los medios digitales. Lo que implica, por ejemplo, tener que potenciar la investigación y análisis de las redes sociales desde una perspectiva más social, más antropológica, estudiando los usos, costumbres, deseos, modos de vida, socialización, etc., situación que podría ser el camino para encontrar la piedra filosofal de la rentabilidad económica del periodismo y los medios. De ahí que resulta interesante aplicar el método abordado por Christine Hine (2004), quien sostiene que el agente de cambio no es la tecnología en sí misma, sino los usos y la construcción de sentido alrededor de ella, razón por la cual existe un espacio de estudio sobre las prácticas cotidianas en torno a internet:

Una etnografía de Internet puede observar con detalle las formas en que se experimenta el uso de una tecnología. En su forma básica, la etnografía consiste en que un investigador se sumerja en el mundo que estudia por un tiempo determinado y tome en cuenta las relaciones, actividades y significaciones que se forjan entre quienes participan en 
los procesos sociales de ese mundo. El objetivo es hacer explícitas ciertas formas de construir sentido de las personas, que suelen ser tácitas o que se dan por supuestas. El etnógrafo habita en una suerte de mundo intermedio, siendo simultáneamente un extraño y un nativo. Ha de acercarse suficientemente a la cultura que estudia como para entender cómo funciona, sin dejar de mantener la distancia necesaria para dar cuenta de ella. (Hine, 2004, p. 18)

La etnografía, en este orden de cosas, puede servir para alcanzar un sentido enriquecido de los significados que va adquiriendo la tecnología en las culturas que la alojan o que se conforman gracias a ella. Y los medios y redes sociales son manifestaciones de esas culturas alojadas en las plataformas de internet.

De este modo, algunas interrogantes que se desprenden a partir de nuestra revisión teórica y que pretendemos explorar, son:

- ¿Cómo los usuarios llegan a comprender las capacidades y posibilidades de internet y las redes sociales? ¿Qué implicaciones tiene su uso? ¿Qué interpretan de ella en tanto medio de comunicación y a quién perciben como audiencia?

- ¿Cuáles son las consecuencias de la información a través de las redes sociales cuya autoría y autenticidad no se ha constatado?

- ¿Es "lo virtual” experimentado como algo radicalmente diferente y separado de "lo real”? ¿Hay una frontera divisoria entre la vida online y offline?

\section{Métodos y fuentes en el estudio de la audiencia en internet}

Con los antecedentes descritos, una línea de investigación que abre diversos campos de análisis en la comunicación mediada por ordenador se produce a través de la etnografía aplicada a la virtualización de la audiencia a través de las redes sociales y su impacto en el periodismo online o ciberperiodismo. Según Nicole Etchevers (2010), la respuesta se da mediante un análisis cualitativo de la presencia y formas de comunicación de los usuarios de medios sociales en internet, en situaciones de relación interpersonal o de comunidades online. Por otro lado, se debe tener en cuenta la importancia de los escritos de los usuarios que pasan a formar parte de las fuentes documentales, lo que, según Alonso Estrada- 
Cuzcano (2016, p. 106), “determina su valor y establece una clasificación desde distintos criterios determinados por el propio investigador".

En dicho contexto, nuestro objeto de estudio son los usuarios de las redes sociales en internet como parte de la audiencia de los medios periodísticos digitales. $\mathrm{Y}$ considerando que internet representa un lugar donde se gesta una cultura, el ciberespacio, establecemos allí nuestro punto de partida. El segundo componente del estudio es la comunicación mediada por ordenador (CMC, Computer-Mediated Communicactions), lo que nos lleva a realizar un esfuerzo experimental, intentando entender los hábitos y costumbres de las personas usuarias de redes sociales, puesto que es el leitmotiv de los medios, empecinados en encontrar el modelo de negocio en entornos del ciberespacio.

Para llevar a cabo esta metodología cualitativa, se ha considerado la alternativa etnográfica por ser un método de trabajo que describe directamente las múltiples formas de vida de los seres humanos, mediante la observación, participación y descripción comprensiva de lo que la gente hace, cómo se comporta y cómo interactúan entre sí.

Se debe tener en cuenta que la metodología etnográfica no queda ajena al contexto en que se desarrolla, y por eso se considera desde una perspectiva adaptativa que reflexiona precisamente alrededor del método. De esta forma, en la medida que el investigador avance, conozca y experimente su problema de estudio, surgen nuevas opciones de investigación que le descubren opciones que en un principio puede no haber tenido previstas (Hine, 2004, p 35-36).

Para Juan Andrés Nadruz (2010), abordar un estudio etnográfico de las redes sociales virtuales requiere de una labor metodológica aplicada específicamente para una etnografía online, por lo que se hace necesario configurar la investigación en tres etapas diferentes y correlativas, con motivo de permitir un alcance y precisión más clara y concisa a los objetivos trazados, enfocándose desde lo general a lo particular, desde lo diferido a lo directo, desde lo abarcado a lo puntual. Así, las técnicas etnográficas aplicadas para este tipo de estudio pueden ser: i) observación flotante, ii) observación diferida y iii) entrevista semiestructurada online a través de un canal de chat. 
Con estas técnicas, vemos que la investigación sobre la audiencia digital en espacios virtuales debe basarse en una metodología cualitativa que presente su fundamentación en el trabajo de campo desarrollado, en el cual se ha logrado un acervo de evidencia documental y entrevistas mediante la observación participante en el contexto.

\section{Antropología y etnografía del ciberespacio}

Ángel Díaz de Rada afirma que

[...] la investigación antropológica de las formas del saber humano es hoy una tarea que no ha de centrarse solamente en las categorías del pensamiento o en las formas del lenguaje como procesos relativamente autónomos, sino, también, las formas de práctica que las personas ponen en juego en concretos escenarios de acción social. [...] la cuestión, hoy, no es ya tanto ¿qué forma tiene una estructura del saber?, como ¿qué hacen las personas en su vida ordinaria con sus saberes?, ¿cómo se configuran estos (saberes) en el contexto de sus intereses y ejercicios prácticos? $(2010$, p. 143)

Por tanto, creemos que la Antropología puede - y debe - guardar estrecha relación con las tecnologías digitales, en donde se mueven grupos focales diseminados en distintas redes sociales.

Las técnicas de investigación de la antropología y de la etnografía, con su característica de inmersión en la sociedad en red para un estudio de los usos, costumbres y hábitos que cambian la vida de las personas, son elementos importantes en el campo de estudio del ciberperiodismo y los nuevos medios. Diversos investigadores coinciden en afirmar que, para ayudar a la supervivencia de los medios, hay que hacer investigación de calidad apoyándose en la multidisciplinariedad de las ciencias del conocimiento.

Si bien es cierto que la antropología y la etnografía son disciplinas del siglo pasado, sin embargo, al igual que otras disciplinas del conocimiento han evolucionado encontrándose con nuevos modos de actuar en un nuevo ecosistema virtual de personas que tienen sus propias características y necesidades. Mientras 
lo digital ha sido abordado por la antropología (Escobar, 2005; Horst \& Miller, 2012) y existen ya numerosas investigaciones de corte etnográfico que abordan lo digital como campo de estudio (Coleman, 2010), son mucho más escasos los trabajos que pretenden afrontar el cruce entre digitalidad y visualización (Estalella \& Ardèvol, 2007; Pink \& Horts, 2016). Así, nos preguntamos, por ejemplo: ¿de qué maneras operan las tecnologías digitales de la información en relación con la conformación de identidades y de la noción misma de diferencia? ¿De qué formas intervienen en los procesos de exclusión social y cuáles son los alcances de estas para facilitar la participación inclusiva de grupos culturalmente diversos? ¿Cuáles son los actores, discursos y agendas implicados en estos procesos? ¿Cómo se produce la democratización del acceso y la restitución del tejido social en internet? E igualmente importante, ¿cómo sucede, en y con lo digital, la instrumentalización de la diversidad? La brecha Norte-Sur ¿ha pasado a ser una brecha únicamente digital? ¿Cómo se vincula este proceso con la regularización de distintos procesos sociales? ¿Cómo incluir las tecnologías digitales en la investigación sobre la diversidad cultural? ¿Cómo se relacionan la virtualidad con nuevas formas de visualización? La respuesta conjunta a estas variables es rotunda: es necesario indagar en los procesos de mediatización, alfabetización, visualización y espectacularización de lo cultural, ya que estos son la expresión de distintas transformaciones sociales y espacios potenciales para la acción social.

Por tanto, la antropología y la etnografía, así como su relación con las tecnologías digitales emergentes y la expansión y crecimiento de comunidades virtuales, deben constituirse en líneas y proyectos de investigación fundamentales para aplicarlos al campo de la comunicación y el periodismo.

\section{Ciberantropología de las redes sociales}

El profesor de periodismo de la Universidad de la Ciudad de Nueva York (CUNY, por sus siglas en inglés), Jeff Jarvis, citado por Pisani (2008), propone la noción de "periodismo reticular" que toma en cuenta la naturaleza colaborativa del periodismo de hoy. Profesionales y aficionados trabajan juntos para sacar lo mejor. Establecen enlaces independientemente de los medios para los cuales trabajan, 
pueden ignorar las fronteras tradicionales. Comparten hechos, preguntas, respuestas, ideas y perspectivas. El periodismo reticular es asunto de proceso tanto como de producto. Según Pisani:

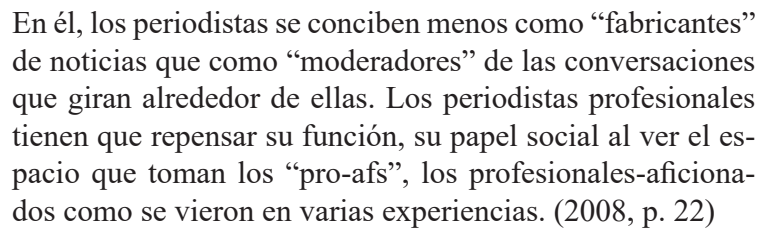

El ecosistema de los medios sigue cambiando y transformándose, en la medida en que surgen nuevas tecnologías de la información que provocan una disrupción en las organizaciones de noticias, en los profesionales y en la esencia del periodismo y de sus procesos informativos y comunicativos.

Por otra parte, los usuarios de redes sociales en internet dan sentido a sus prácticas a través de una comprensión compartida, que surge tanto de la producción de una página web como del uso de un grupo de noticias, y que constituyen nada menos que formas de acción social (Hine, 2004). Una evolución natural ha sido la creación de grupos de noticias que no son más que espacios sociales altamente diferenciados, sostenidos por colaboraciones de usuarios en forma de notas y mensajes temporales y situacionalmente relevantes para una comunidad en internet.

En dicho contexto, la pregunta que surge es: ¿qué investigamos de las redes sociales? ¿Interesa investigar el software o la interfaz de la red social? O, por el contrario, ¿interesa investigar el uso que se da a través de esas interfaces? Considerando que son individuos o conjuntos de estos los que están detrás de dichas interfaces de redes sociales, ¿es interesante para el medio que es lo que hace interactuar al usuario de cara a un mensaje o artículo periodístico?, ¿cuáles son sus necesidades?, ¿qué opinan sobre tal o cual tema?

Para responder a estas variables se han abordado investigaciones desde perspectivas más sociológicas y económicas. Pero se ha dejado de lado el estudio de los usuarios desde su perspectiva más antropológica y etnográfica. De hecho, cada vez más investigaciones sobre internet y redes sociales se están constituyendo en un ámbito de enorme interés etnográfico y antropológico (Contreras, 2004, 
p. 53). En esta línea, la pregunta que subyace es: ¿por qué ahora el interés por este entorno? La respuesta es simple pero contundente: porque necesitamos saber cuáles son los usos, costumbres, deseos, necesidades, creación, opiniones, contextualización, etc., de los usuarios, quienes, formando comunidades virtuales, intercambian mensajes, también, a través de plataformas virtuales.

\section{Etnografía virtual o digital}

Antes de adentrarnos en el mundo de la etnografía virtual propuesto por Christine Hine (2004), es necesario dejar claro el concepto de la etnografía en sus orígenes. La etnografía tiene sus orígenes en la antropología y la sociología. Anthony Giddens y Philip Sutton (2014), la definen como el estudio directo de personas o grupos durante un cierto periodo, utilizando las técnicas de la observación participante o las entrevistas para conocer su comportamiento social. Etnografía significa literalmente: descripción de un pueblo. Por tanto, la etnografía se ocupa de las personas en sentido colectivo, no de los individuos. Así, es una manera de estudiar a las personas en grupos organizados duraderos a los que cabe referirse como comunidades o sociedades (Angrosino, 2012, p. 19). La etnografía es, ante todo, una actividad investigativa de corte descriptivo que pretende estudiar la cultura de una comunidad. Por tanto, la etnografía es un método de investigación por el que se aprende el modus vivendi de una unidad social concreta, pudiendo ser esta una familia, una clase social o una escuela.

Esta definición casa con los objetivos de las organizaciones que viven del ofrecimiento de productos y servicios a comunidades de usuarios. De ahí que estudiar comunidades es el objetivo de las organizaciones de noticias, instituciones y corporaciones. Pero es un tipo de comunidades propias y emergentes de este milenio, que se desarrollan a través de interfaces de comunicación mediadas por ordenador (Computer-Mediated Commmunications, CMC), conocidas como comunidades virtuales. En esta línea, Joseph Dumit (2014, p. 350) habla de implosión de proyectos, que define como los intentos de enseñar y aprender acerca de la incrustación de objetos, hechos, acciones y personas en el mundo y el mundo en ellos. 
Independientemente de los diversos alcances que puede tener la técnica etnográfica, según el objeto de estudio de cada investigador, se desprenden algunas pautas (cuadro 1) con el objetivo de comprender e interpretar una realidad que interactúa en un contexto más amplio, cuya finalidad es obtener conocimientos y planteamientos teóricos más que resolver problemas prácticos como lo podría hacer la investigación-acción.

Cuadro 1. Técnicas etnográficas

\begin{tabular}{|l|l|}
\hline \multicolumn{2}{|c|}{$\begin{array}{r}\text { ESPECTRO DE METODOLOGÍAS ETNOGRÁFICAS } \\
\text { EN AMBIENTES VIRTUALES }\end{array}$} \\
\hline Observación & Puede ser interactiva, directa y participante \\
\hline Exploración con profundidad in situ & $\begin{array}{l}\text { Inmersión en el trabajo de campo (ejemplo: } \\
\text { en casa) }\end{array}$ \\
\hline Documentación & Fotografía, video, audio \\
\hline Diarios & Blogs, sites, collages \\
\hline $\begin{array}{l}\text { Uso de técnicas móviles de grabación } \\
\text { (entrevistas y cuestionarios) }\end{array}$ & $\begin{array}{l}\text { Por ejemplo, smartphones para seguir } \\
\text { el comportamiento del usuario }\end{array}$ \\
\hline
\end{tabular}

Fuente: Angrosino, 2012. Elaboración propia.

Para Natalie M. Underberg y Elayne Zorn (2014), la etnografía digital puede entenderse como un método para representar culturas de la vida real a través de la narración en medios digitales. Haciendo que las audiencias vayan más allá de absorber hechos, la narración computarizada permite la inmersión en la experiencia de otra cultura. Es una guía para cualquier persona en las ciencias sociales que busca enriquecer técnicas etnográficas, la etnografía digital ofrece un enfoque innovador que utiliza componentes interactivos para simular narrativas culturales. Sarah Pink y Heather Horst (2016), coautores de Digital Ethnography, uno de los pocos libros en su campo para abordar la brecha digital entre los investigadores, aborda el extraordinario potencial de enriquecimiento ofrecido por los recursos tecnológicos, muy lejos de restringir la investigación a los métodos cuantitativos usualmente asociados con la tecnología. 
Partimos de la situación actual, en donde cada vez más personas están accediendo a noticias a través de teléfonos móviles como nunca antes se había producido con un medio masivo, abriendo una oportunidad para las organizaciones de noticias que quieren y necesitan explorar nuevas formas de atraer audiencias y satisfacer las cambiantes necesidades de información. Para ello, hay que desarrollar formas efectivas de involucrar a las audiencias en el acceso a las noticias móviles. Sin embargo, las organizaciones de noticias deben entender mejor el comportamiento del usuario y las tendencias. Un reciente estudio desarrollado por la Fundación Knight en colaboración con Nielsen, destaca la creciente importancia del teléfono móvil para los proveedores de noticias, ya que casi nueve de cada diez personas utilizan este aparato para acceder a las mismas, a lo que se suma el creciente papel de las redes sociales cuyo objetivo se ha convertido en llevar esas noticias a la gente.

\section{Materiales empleados: estudio de audiencias móviles desde una perspectiva ciberantropológica}

Los rápidos avances en tecnología han dejado a las organizaciones de noticias en un escenario de lucha por administrar cómo se crean, consumen y entregan las noticias, pero la gente ha cambiado la forma de acceder a ellas. Antes, lo hacían - y aún lo hacen- a través de ordenadores de sobremesa y portátiles, y ahora —de forma creciente-, mediante el omnipresente teléfono inteligente. Según el informe de 2016, News Goes Mobile: How People Use Smartphones to Access Information, de la Knight Foundation y elaborado por la consultora Nielsen, indica que desde 2011, la tasa de posesión de teléfonos inteligentes para adultos en los EE. UU. ha aumentado notablemente de 46 a $82 \%$, acercándose a un punto de saturación entre algunos grupos de edad. En los últimos dos años, el consumo individual de noticias móviles ha crecido rápidamente. De hecho, el $89 \%$ de la población móvil estadounidense (144 millones de usuarios) actualmente tiene acceso a noticias e información a través de dispositivos móviles.

A medida que las organizaciones de noticias tratan de administrar mejor esta transformación digital de plataformas, así como interactuar con su audiencia y 
mantenerse competitivas, surgen dos grandes interrogantes: i) ¿qué deberían entender los medios sobre el comportamiento cambiante de su audiencia en el acceso a las noticias móviles?; y, ii) ¿de qué forma las distintas audiencias acceden a las noticias e información a través de los teléfonos móviles?

El estudio profundiza en varios hallazgos claves y luego se sumerge en la forma como grupos específicos de personas utilizan diferentes plataformas móviles para acceder a las noticias, por lo que los resultados arrojan la siguiente lectura:

1. Hay una audiencia considerable que consume noticias en teléfonos móviles. Casi toda la población de usuarios adultos de móviles consume noticias en sus dispositivos y cada vez más usuarios pasan el tiempo accediendo a noticias a través de las plataformas sociales. Concretamente, 144 millones de usuarios actualmente tienen acceso a noticias e información a través de dispositivos móviles (figura 1).

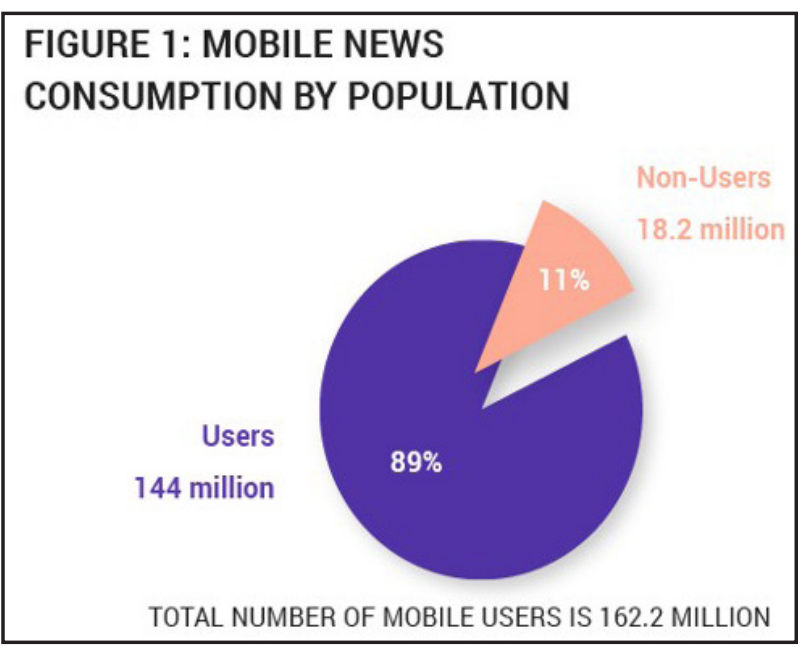

Fuente: Foundation, 2016.

2. Los usuarios de teléfonos móviles solo gastan, en promedio, el $5 \%$ del tiempo de su uso del móvil en las noticias; ello incluye noticias "duras" sobre eventos actuales y noticias globales, en contraposición a informes meteorológicos rutinarios y otras formas de noticias "blandas" (figura 2). De hecho, el $27 \%$ del 
tiempo de uso del móvil (más de 12 horas al mes) se gasta en sitios de redes sociales. Así, la encuesta complementaria de Nielsen mostró que la mitad de los usuarios de redes sociales pasan tiempo buscando noticias. E1 $70 \%$ de los usuarios de Facebook, por ejemplo, usa esta red social para el acceso a las noticias todos los días.

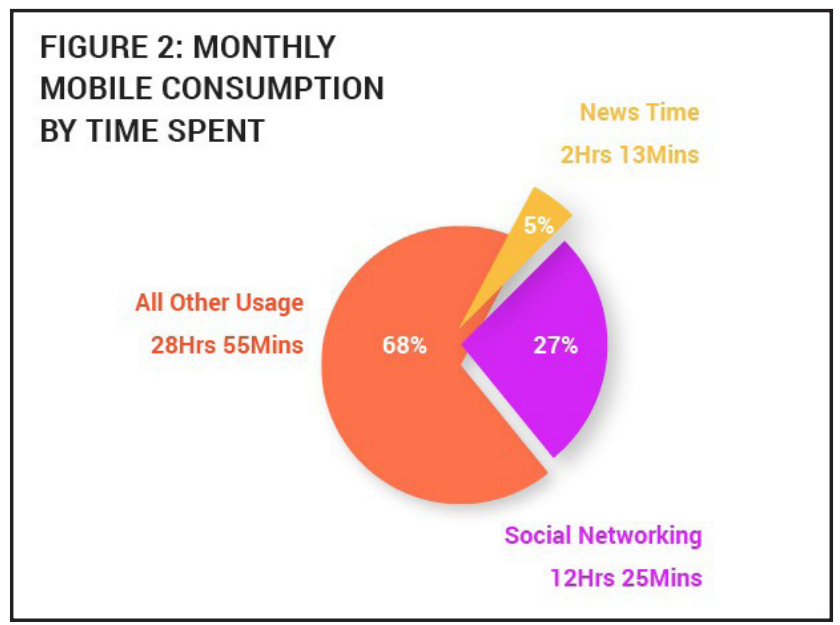

Fuente: Knight Foundation, 2016.

3. Los usuarios de teléfonos móviles que acceden a las noticias a través de aplicaciones pasan más tiempo leyendo el contenido, pero esa audiencia que usa aplicaciones es pequeña, por lo que es esencial saber quiénes son esos usuarios.

4. Los sitios y aplicaciones de medios sociales son fuentes importantes de noticias para los usuarios de redes sociales, aunque la televisión sigue siendo su principal fuente. Sin embargo, un análisis de los cambios interanuales (figura 3) sugiere que el tiempo dedicado directamente a las aplicaciones y sitios móviles ha disminuido en el último año. Esta disminución contrasta con la actividad sustancial de noticias que tiene lugar en las plataformas de redes sociales. Esto es así porque los usuarios de las redes sociales también dependen de amigos, contactos y personas que siguen como fuentes de noticias de confianza tanto como, o más, de los que dependen de los medios de comunicación. 
FIGURE 3: MONTHLY MOBILE NEWS USAGE 2013/14 VS. 2014/15

News Category (Web + Apps)

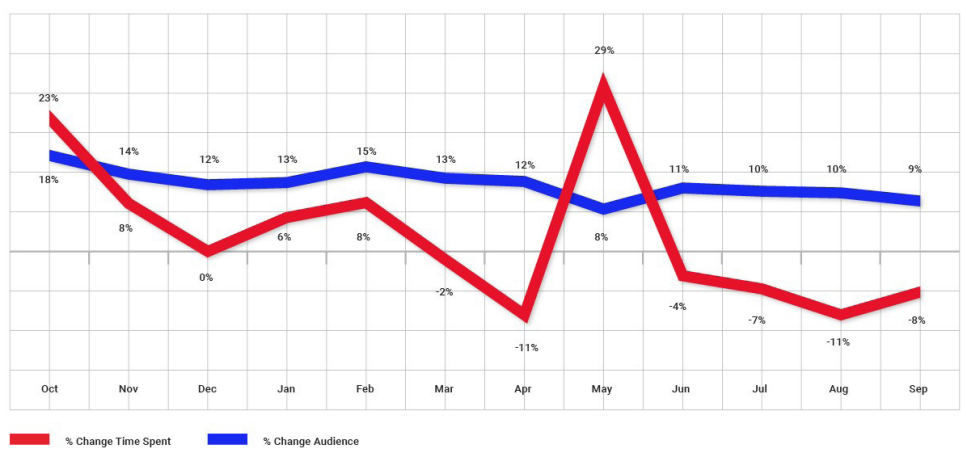

Tomado de: Knight Foundation, 2016.

5. Los usuarios de noticias en teléfonos móviles, activos en las redes sociales, no solo se involucran de forma pasiva con el contenido de noticias, sino que toman acciones sin conexión relacionadas con el contenido.

Aunque el estudio focaliza su universo en la población estadounidense, es fácilmente extrapolable a otras regiones en donde impera la sociedad red. Así, mientras que las organizaciones de noticias, de alcance nacional e internacional, están experimentando y se mantienen activas en plataformas móviles y en las redes sociales, muchos periódicos locales y regionales han sido más lentos en su migración hacia las capacidades necesarias para convertirse en operaciones del tipo "lo digital primero" (cuyo contenido desarrollado prioriza la producción para plataformas móviles y en línea sobre la del producto impreso). Incluso los grandes editores y los periódicos locales en línea están luchando con la forma de distribuir contenido digital a través de canales sociales, como los artículos instantáneos de Facebook, con el fin de aprovechar al máximo los posibles ingresos de esta gran audiencia.

Sin embargo este estudio, con toda la riqueza de datos aportados en donde se conjugan elementos como las personas (con sus usos y costumbres) y el lugar 
territorial (interfaces virtuales), debe tener un siguiente paso en su elaboración: la aplicación de la antropología del ciberespacio (ciberantropología) y la etnografía virtual.

Es evidente que en la época en la que nos encontramos, para la aplicación de los estudios antropológicos y etnográficos en donde los conceptos de globalización y tecnologías están estrechamente relacionados, hablar de fronteras resulta un tanto utópico.

Por ejemplo, podemos partir de las necesidades de información de los usuarios y elaborar un mapa de conocimiento antropológico. Como herramienta metodológica que puede servir de indicador de lo que los usuarios quieren, se plantea recurrir a las palabras más buscadas en internet (cuadro 2), utilizando el buscador Google, y que han marcado una tendencia durante un periodo de tiempo que puede ser una semana, un mes o un año. Cada palabra o descriptor nos proporciona una pista importante para iniciar el punto de partida en la investigación de la audiencia en internet, utilizando las técnicas antropológica y etnográfica descritas.

\section{Cuadro 2. Temas de ámbito internacional que han alcanzado máximos históricos en interés de búsqueda en 2016}

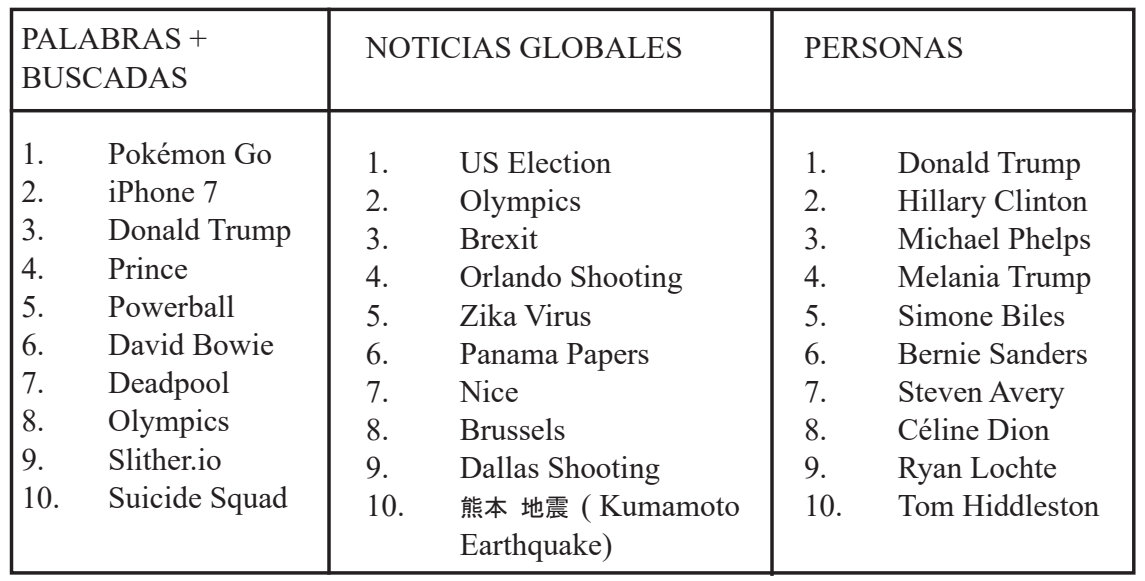




\begin{tabular}{|c|c|c|}
\hline $\begin{array}{l}\text { PALABRAS + } \\
\text { BUSCADAS }\end{array}$ & $\begin{array}{c}\text { EVENTOS DEPORTIVOS } \\
\text { GLOBALES }\end{array}$ & $\begin{array}{c}\text { PERSONALIDADES } \\
\text { DESAPARECIDAS } \\
\text { (FALLECIDAS) }\end{array}$ \\
\hline $\begin{array}{ll}\text { 1. } & \text { iPhone } 7 \\
\text { 2. } & \text { Freedom } 251 \\
\text { 3. } & \text { iPhone SE } \\
\text { 4. } & \text { iPhone 6S } \\
\text { 5. } & \text { Google Pixel } \\
\text { 6. } & \text { Samsung Galaxy S7 } \\
\text { 7. } & \text { iPhone 7 Plus } \\
\text { 8. } & \text { Note } 7 \\
\text { 9. } & \text { Nintendo Switch } \\
\text { 10. } & \text { Samsung J7 }\end{array}$ & $\begin{array}{ll}\text { 1. } & \text { Rio Olympics } \\
\text { 2. } & \text { World Series } \\
\text { 3. } & \text { Tour de France } \\
\text { 4. } & \text { Wimbledon } \\
\text { 5. } & \text { Australian Open } \\
\text { 6. } & \text { EK } 2016 \\
\text { 7. } & \text { T20 World Cup } \\
\text { 8. } & \text { Copa América } \\
\text { 9. } & \text { Royal Rumble } \\
\text { 10. } & \text { Ryder Cup }\end{array}$ & $\begin{array}{ll}\text { 1. } & \text { Prince } \\
\text { 2. } & \text { David Bowie } \\
\text { 3. } & \text { Christina Grimmie } \\
\text { 4. } & \text { Alan Rickman } \\
\text { 5. } & \text { Muhammad Ali } \\
\text { 6. } & \text { Leonard Cohen } \\
\text { 7. } & \text { Juan Gabriel } \\
\text { 8. } & \text { Kimbo Slice } \\
\text { 9. } & \text { Gene Wilder } \\
\text { 10. } & \text { José Fernández }\end{array}$ \\
\hline
\end{tabular}

Fuente: Google Trends. Recuperado de: https://www.google.com/trends/yis/2016/GLOBAL?hl=es

\section{Resultados y discusión}

Cada vez más investigadores y laboratorios de medios tienen como meta el estudio de la audiencia y su relación con las tecnologías emergentes de la información, como es el caso del internet Media Lab de la Universidad Complutense de Madrid o del Medialab de la Universidad de San Marcos y que, según OyarceCruz (2017, p. 187), “además de la difusión mediante podcast y streaming, está enfocada en proyectos de investigación interdisciplinaria, en la convergencia multimedia y de uso y aplicación de tecnología para mejorar la comunicación e información".

En dicho contexto, el estudio de la nueva audiencia digital y móvil que se da a través de las redes sociales en internet (usuarios de dispositivos móviles), se inscribiría en el contexto de la denominada "antropología o etnografía del ciberespacio". Algunos autores como Pau Contreras (2004) creen más apropiado hablar de antropología o etnografía en el ciberespacio, puesto que las practicas objeto del estudio se desarrollan casi íntegramente en la red. No obstante, los temas a través de las interacciones que se dan, fundamentalmente, a través de las redes sociales forman parte de categorías mucho más generales, como los movimientos 
sociales o la construcción de conocimiento, que se extiende más allá del ciberespacio, "hasta los territorios que han sido los tradicionales objetos de estudio de la disciplina antropológica".

Estudios como el de Meyer (1996) o Paccagnella (1999) citados por Contreras (2004, p. 56), aportan pistas interesantes respecto a la observación en entornos virtuales y la validez de los datos recogidos. Por su parte, Christine Hine (2004) sostiene que internet permite un estudio detallado de las relaciones en línea, de modo que la red no es solo un medio de comunicación, sino también un artefacto cotidiano en la vida de las personas y un lugar de encuentro que permite la formación de comunidades, de grupos más o menos estables; en definitiva, lo que debemos estudiar con profundidad es la emergencia de una nueva forma de sociedad o sociedad red.

Así pues, dado que en los trabajos de los teóricos nombrados se proponen metodologías que a juicio de cada uno pueden ser las más apropiadas, hemos considerado que para el estudio de la audiencia con cultura de red, concretamente, para las comunidades de usuarios de redes sociales que, directa o indirectamente, participan en la consolidación del ciberperiodismo, proponemos las siguientes técnicas de investigación etnográfica (cuadro 3), tanto cualitativas como cuantitativas.

\section{Cuadro 3. Técnicas de investigación etnográfica para el} estudio de la ciberaudiencia

\begin{tabular}{|l|l|l|}
\hline \multicolumn{2}{|l|}{ TÉCNICAS DE INVESTIGACIÓN ETNOGRÁFICA } \\
\hline & $\begin{array}{l}\text { La observación es el acto de percibir las actividades e inte- } \\
\text { rrelaciones de las personas en el entorno de campo median- } \\
\text { te los cinco sentidos de la investigación (Angrosino, 2012, } \\
\text { p. 61). Por tanto, no cabe duda de que una parte importante } \\
\text { de las investigaciones se da a través de este método, dado } \\
\text { que permite al investigador hacer una inmersión en el obje- } \\
\text { to de estudio a través de la observación directa y participan- } \\
\text { te. Para ello, el investigador debe establecer contacto con } \\
\text { distintas plataformasión } \\
\text { de comunidades } \\
\text { sociales virtuales. } \\
\text { 2. Entrevistas }\end{array}$ & $\begin{array}{l}\text { las comunidades virtuales de redes sociales, previo registro } \\
\text { de la plataforma elegida. En esta fase, en la que se asigna un } \\
\text { cronograma de duración en el espacio-tiempo, se establece } \\
\text { contacto directo con los usuarios, por ejemplo, de un medio } \\
\text { o periódico, a través de mensajes que pueden etiquetarse, }\end{array}$ \\
\hline
\end{tabular}




\begin{tabular}{|c|c|c|}
\hline \multirow[t]{2}{*}{$\begin{array}{l}\text { TÉCNICAS } \\
\text { CUALITATIVAS }\end{array}$} & $\begin{array}{l}\text { mediadas por } \\
\text { ordenador (o por } \\
\text { teléfono) y de forma } \\
\text { presencial con la } \\
\text { muestra de la } \\
\text { población } \\
\text { seleccionada. }\end{array}$ & $\begin{array}{l}\text { acción que redunda en un conocimiento de ciertas ca- } \\
\text { racterísticas de los usuarios móviles de las redes sociales } \\
\text { por las afinidades o diferencias que se pueden descubrir. } \\
\text { La entrevista es un proceso por el que se dirige una con- } \\
\text { versación para recoger información (Angrosino, 2012, } \\
\text { p. 66). Para los casos de investigación del periodismo } \\
\text { relacionados con las redes sociales, una vez hecha la } \\
\text { selección de las personas a entrevistar (tanto de usua- } \\
\text { rios como responsables del área de redes sociales de un } \\
\text { medio) esta técnica etnográfica permite saber de modo } \\
\text { directo la opinión de los distintos actores del medio en } \\
\text { internet (cibermedio) o del periodismo en red (ciber- } \\
\text { periodismo). Por tanto, las entrevistas han de ser tanto } \\
\text { cerradas como abiertas. Para las entrevistas mediadas } \\
\text { por ordenador (CMC), pueden utilizarse los siguientes } \\
\text { canales: correo electrónico, chat privado, lista de distri- } \\
\text { bución o incluso la propia comunidad de red social, cuya } \\
\text { característica interna disponga de un modelo de mensa- } \\
\text { jería instantánea. Todo esto puede ser complementado } \\
\text { con las entrevistas del modelo "cara a cara" (Face To } \\
\text { Face o presencia física). }\end{array}$ \\
\hline & $\begin{array}{l}\text { 3. Investigación de } \\
\text { archivos o fuentes } \\
\text { primarias. }\end{array}$ & $\begin{array}{l}\text { La investigación de archivos es el análisis de materiales } \\
\text { que se han almacenado para investigación, servicio y } \\
\text { otros propósitos tanto oficiales como no oficiales (An- } \\
\text { grosino, 2012, p. 74). Algunos etnógrafos prefieren lla- } \\
\text { marlos fuentes primarias. En el análisis de las comuni- } \\
\text { dades virtuales y de redes sociales, las fuentes primarias } \\
\text { las constituyen, en primer lugar, la bibliografía consul- } \\
\text { tada que permite plantear el marco teórico y conceptual } \\
\text { con los antecedentes de la investigación. Adicionalmen- } \\
\text { te a la bibliografía, el investigador puede optar por pre- } \\
\text { sentar como fuente primaria el análisis de las publica- } \\
\text { ciones, tutoriales, aplicaciones propietarias (programas } \\
\text { de software), guías o manuales de estilo asociadas a las } \\
\text { redes sociales que las organizaciones de noticias puedan } \\
\text { ya tener como la hoja de ruta a seguir en el desarrollo de } \\
\text { los contenidos informativos. }\end{array}$ \\
\hline \multirow{4}{*}{$\begin{array}{l}\text { TÉCNICAS } \\
\text { CUANTITATIVAS }\end{array}$} & \multirow{4}{*}{$\begin{array}{l}\text { Encuestas, cues- } \\
\text { tionarios y censos. } \\
\text { (Tienen como } \\
\text { objetivo identificar } \\
\text { tendencias en las } \\
\text { conductas.) }\end{array}$} & $\begin{array}{l}\text { Encuestas cara a cara: Consisten en entrevistas direc- } \\
\text { tas y personales con cada encuestado que forma parte de } \\
\text { la muestra seleccionada. }\end{array}$ \\
\hline & & $\begin{array}{l}\text { Encuestas telefónicas: Este tipo de encuesta se basa, } \\
\text { previa muestra seleccionada, en una entrevista telefóni- } \\
\text { ca con cada encuestado. }\end{array}$ \\
\hline & & $\begin{array}{l}\text { Encuestas por correo electrónico: Consiste en el envío } \\
\text { de un cuestionario a los potenciales encuestados, pedir- } \\
\text { les que lo rellenen y lo devuelvan completado. }\end{array}$ \\
\hline & & $\begin{array}{l}\text { Encuestas online: Este tipo de encuesta consiste en } \\
\text { colocar un cuestionario en una página web o crear una } \\
\text { encuesta en internet y enviarla a los correos electrónicos } \\
\text { (Google proporciona una plataforma para este modelo). }\end{array}$ \\
\hline
\end{tabular}




\section{Conclusiones}

Aunque resulte una obviedad, estudiar a las comunidades online que se generan a través de las plataformas móviles y usuarios de redes sociales es hacer referencia obligada al estudio de las particularidades del espacio virtual. Afortunadamente, es latente el crecimiento de científicos pioneros en propuestas metodológicas, como los tratados en este trabajo, cuyos planteamientos ayudan a definir la aproximación y las técnicas que se deben usar en proyectos de investigación sobre usuarios en internet (audiencia digital) y la construcción del conocimiento para fortalecimiento del ciberperiodismo (o periodismo en red).

El papel del investigador que utiliza las técnicas ciberantropológicas o etnográficas en el estudio de las audiencias digitales no solo permite acercarse a una realidad para describirla de manera detallada, sino que requiere que el investigador se introduzca en esta interacción mediada por ordenador.

De ahí que el camino que se ha de seguir en el estudio cualitativo de la audiencia digital de los medios debe tener un fundamento etnográfico y neurocientífico puesto que la investigación del periodismo en internet (ciberperiodismo) se abre a otras fuentes utilizando técnicas de las ciencias sociales. Este nuevo entorno de la investigación acrecienta el hecho de que la construcción del nuevo periodismo requiere de la imbricación de otras ciencias disciplinares, lo que redundará en beneficio de las organizaciones de noticias, de la sociedad como parte activa en el proceso de construcción de la información, de los profesionales, de los académicos y de la propia disciplina del periodismo.

La pretensión de este trabajo ha sido indicar cómo marcar la ruta etnográfica a utilizar como muestra de una vía eficaz en el estudio de audiencias y de las tendencias comunicativas, aportando riqueza a la investigación de las comunicaciones mediadas por ordenador. Por ello, tanto en las comunidades técnico-científicas como en las ciencias sociales han comenzado a conformarse nuevas perspectivas de investigación; estas incorporan otras disciplinas del conocimiento como las descritas junto con las tecnologías emergentes o disruptivas con el objetivo de dar respuestas a las nuevas variables como las que aquí planteamos. En estas últimas, un campo completo de enseñanza e investigación debe tomar forma alrededor de 
la propuesta del programa de estudios de ciencia, tecnología y sociedad, el mismo que debe ser institucionalizado en las facultades de Periodismo y Comunicación.

\section{Referencias bibliográficas}

Angrosino, M. (2012) Etnografía y observación participante en investigación cualitativa. Madrid: Ediciones Morata.

Brown, M. F. (2010). Relativismo cultural 2.0. En F. Cruces \& B. Pérez (Comps.), Textos de Antropología Contemporánea (pp. 25-57). Madrid: Universidad Nacional de Educación a Distancia.

Coleman, G. (2010). Ethnographic approaches to digital media. Annual Review of Anthropology, 39, 487-505. Recuperado de http://www.annualreviews.org/doi/abs/10.1146/annurev.anthro.012809.104945?journalCode $=$ anthro.

Contreras, P. (2004). Me llamo Kohfam. Identidad hacker: una aproximación antropológica. Barcelona: Gedisa.

Cremades, J. (2001). El paraíso digital. Claves para entender la revolución de Internet y las Telecomunicaciones. Barcelona: Plaza \& Janés.

Diaz de Rada, A. (2010) Etnociencia. El orden del sentido y el sentido del orden. En F. Cruces \& B. Pérez (Comps.), Textos de Antropología Contemporánea (pp. 143-177). Madrid: Universidad Nacional de Educación a Distancia.

Dumit, J. (2014). Writing the Implosion: Teaching the World One Thing at a Time. Cultural Anthropology 29( 2), 344-362.

Escobar, A. (2005). Bienvenidos a Cyberia. Notas para una antropología de la cibercultura. Trad. Carlos Barragán. Revista de Estudios Sociales, 22, 15-35.

Estalella, A., \& Ardèvol, E. (2007). Ética de campo: hacia una ética situada para la investigación etnográfica de internet. Forum Qualitative Social Research, 8, 3-8.

Estrada-Cuzcano, A. (2016). Presencia de la Universidad Nacional Mayor de San Marcos en la Bibliotecología Peruana: Estudio historiográfico. Revista Letras, 87(125). Recuperado de http://revista.letras.unmsm.edu.pe/index.php/le/issue/view/19 
Etchevers, N. (2010). Ruta etnográfica para la comprensión de la comunicación on-line. Salamanca: Instituto Universitario de Ciencias de la Educación, Universidad de Salamanca. Recuperado de http://ddd.uab.cat/pub/ $\operatorname{dim} / 16993748$ n1/16993748n1a2.pdf

Flichy, P. (2001). Lo imaginario de Internet. Barcelona: Tecnos Grupo Anaya.

Giddens, A. \& Sutton, P. (2014). Sociología, séptima ed. Barcelona: Alianza Editorial.

Harris, M. (2014). Antropología cultural. Madrid: Alianza Editorial.

Hine, C. (2004). Etnografía virtual. Barcelona: Editorial UOC.

Horst, H. A. \& Miller, D. (2012). Digital Anthropology. Londres: Bloomsbury.

Knight Foundation. (2016). News Goes Mobile: How People Use Smartphones to Access Information. Recuperado de https://medium.com/mobile-firstnews-how-people-use-smartphones-to

Nadruz, J. A. (2010). Las comunidades virtuales Xbox: interacción y socialización en el ciberespacio. Recuperado de http://www.unesco.org.uy/shs/ fileadmin/templates/shs/archivos/anuario2009/Nadruz.pdf

Oyarce-Cruz, J. (2017). Creación de un laboratorio de medios en la Universidad Nacional Mayor de San Marcos-Perú. Revista Letras, 88(127). Recuperado de: http://revista.letras.unmsm.edu.pe/index.php/le/issue/view/26

Pink, S. \& Horst, H. (2016). Digital Ethnography. Reino Unido: Sage Publications Ltd.

Pisani, F. (2008). Ex-audiencia, blogalaxia y periodismo reticular. En J. Flores (Coord.), Blogalaxia y Periodismo en la Red (pp. 21-24). Madrid: Fragua

Sören Romero, A. (2014). Etnografía Virtual en redes sociales: el caso de Facebook. En iS+D Fundación I+S Avanzada. Recuperado de http://blog. isdfundacion.org/2014/12/01/etnografia-virtual-no-2-etnografia-virtualen-redes-sociales-el-caso-de-facebook-hei-man-2008/

Terceiro, J. B. (1996). Sociedad Digital: Del Homo Sapiens al Homo Digitalis. Madrid: Alianza Editorial.

Underberg, N. \& Zorn, E. (2013). Digital Etnography: Anthropology, narrative, and new media. Austin: University of Texas Press.

Vázquez, A. (2008). Ciberantropología. Cultura 2.0. Barcelona: UOC editorial.

Yates, J. (1994). The understanding and Use of Knowledge in Modern Business. Londres: Routledge. 